\title{
An on-line laboratory in sensation and perception
}

\author{
KEITH D. WHITE \\ University of Florida, Gainesville, Florida 32611
}

\begin{abstract}
Three components of a teaching laboratory in perception are described that (1) compare several psychophysical methods for measuring visual illusions, (2) simulate experiments on dark adaptation, and (3) allow participation in auditory demonstrations or experiments. Features of special interest include: interactive use of graphics, software for simulating experiments (such as EXPER SIM, University of Michigan), and use of A/D, D/A, and TTL ports to interface special peripherals.
\end{abstract}

Most of psychology is distinct from other branches of science in that the phenomena we study can also be experienced. In sensory and perceptual psychology this is especially apparent, and the instructor can capitalize upon the students' immediate experiences as a teaching tool. A significant proportion of the lecture courses as well as the labs on sensation and perception incorporate demonstrations of selected perceptual effects. It is not untypical for the students to react with "oohs" and "ahs," and occasionally these demonstrations seem to evoke a "eureka"-like experience.

In traditional laboratories, one major intention is for students to experience sensory phenomena while, at the same time, they are measuring and studying them. It is not surprising, then, that teaching laboratories following a traditional plan may come to resemble a miniature research lab. Because of this, the students must become at least marginally acquainted with a variety of specialized devices used for the generation of various sensory effects.

This traditional plan for a teaching lab in perception carries with it certain disadvantages, particularly if a substantial number of students wish to enroll. To the extent that a traditional perception teaching lab resembles a research lab, the time and expense of setting up the teaching lab is relatively high. Even the simplest sorts of optical or electronic equipment available for use "off the shelf" can easily cost hundreds or thousands of dollars per item. To design a system based on cheaper components is often costly in faculty time and technical support, both for system development and for future maintenance. As a second consideration, one must consider the need for multiplying these expenditures in accord with student enrollments. Insofar as the teaching tools are modeled after research devices, items of equip. ment frequently cannot handle more than a few students at a time. And who will maintain and repair the devices? Who will show students how to use them?

This paper was developed under a Comprehensive Assistance to Undergraduate Science Education grant (NSF78-06025). The views expressed herein are those of the author and do not necessarily reflect those of the National Science Foundation.
At the University of Florida, the numbers of students who might enroll in a perception teaching lab make the traditional approach impractical. Instead, we have been developing teaching labs around a flexible computer system.

One example of our approach involves a set of interrelated lab exercises that acquaint the students with certain visual illusions as well as with psychophysical methods. The use of the interactive graphics displays permits the student to experience and measure such well-known illusions as the Mueller-Lyer or Ponzo illusions. Any one of these can be shown in the broader context of line-length judgments, and each student must provide his or her own repeated judgments of line lengths on at least one occasion. The judgments are collected in the context of a particular method, which can be selected as method of limits, method of constant stimuli, or magnitude estimation with or without modulus. In this way we can simulate one aspect of a real "hands-on" experiment. The student gains an appreciation for certain practical constraints on a psychophysical experiment, such as the tradeoff between number of judgments per session and the ability to keep one's concentration and response criterion constant. By participating in one rather abbreviated psychophysical experiment, the student can recognize this feature fully.

Of course, the same experiences with psychophysical methods serve more specific teaching functions. No student is ever required to make hundreds or thousands of line-length judgments. As soon as an individual has acted as the subject in one more-or-less "hands-on" experiment, he has the opportunity to generate simulated but realistic data for every combination of visual context and psychophysical method that is included in our package. These simulated data can be analyzed by the students for any of several didactic goals, including (1) comparison of point of subjective equality vs. point of objective equality, sometimes called the time-order error, (2) calculation of just-noticeable differences, (3) comparison of the magnitudes of various visual illusions, (4) comparison of the biases inherent in certain types of methods, and (5) statistical analysis 
of the responses under the phi-gamma hypothesis or other proposed models of psychophysical response.

A second group of laboratory exercises rely to a major extent on the simulation of data by the computer. The visual phenomenon of dark adaptation is a prime example because it is a rare student who has not experienced this effect on various occasions in day-today affairs. So there is little need to produce the experience as a laboratory phenomenon. The students instead design simulated experiments that require minutes, rather than days or weeks, to produce the desired results. Here, our resources are focused on teaching students to manipulate variables in "meaningful" ways.

We program into the computer some equations and templates of ideal data for dark-adaptation curves. The software that handles the parameters is essentially the Louisville experimental simulation package. As a supplement to the text readings, the beginning students simulate the effects due to wavelength, retinal location, and so forth, on the course of dark adaptation. These classic sorts of manipulations permit the beginning students to analyze their simulated protocols with regard to differences between rods and cones, or between foveal vs. peripheral retinal areas, and so forth. Higher level students have access to a greater range of variables and can carry out more sophisticated inquiries. Samples of these include the consensual pupillary responses, the Stiles-Crawford effect, the influences of age or gender, as well as changes that might accompany certain disease states or genetic conditions. In this way, the common theme of studying dark adaptation can be applied without difficulty to a fairly wide range of other specific teaching goals.

Lastly, we have a cluster of lab exercises in which it is not only important for students to experience the effect being studied, but it is complex to implement this demonstration, or it requires expensive equipment. A good example is the generation of complex sounds to show auditory phenomena. Now, many research labs produce the complex sounds by a computer, and we naturally thought that a research-like approach might be feasible for the teaching lab. Some insurmountable difficulties were discovered. On our graphics terminals there were too many system interrupts, such as the screen refreshing cycle, which presented unavoidable and undesirable contaminants to the real-time production of auditory stimuli.

We have now opted for an approach that uses the computer's flexibility to program a special-purpose interfacing device. The interface is designed around an integrated-circuit chip intended for the generation of complex sounds. This chip is made by Texas Instruments ${ }^{1}$ but can be obtained from local sources such as Radio Shack. Its main purpose as an interface is to reduce the real-time requirements in programming the computer. One can merely "latch in" parameter values for the frequency, modulation of the frequency and amplitude, and so forth, without requiring the computer to seek out machine language subroutines that operate with sufficient speed.

This special-purpose interface has several features. Some functions can be addressed at TTL levels, a common sort of output on a parallel bus line. Other functions require analog voltages, and these can be provided via $\mathrm{D} / \mathrm{A}$ converters or analog networks. Since it is also possible to provide mutliplex outputs from this circuit at high rates, one can set up a "handshaking" arrangement between the interface and the computer via a logic input line. The advantage of multiplexing is that sounds composed of several frequency bands, like the sounds of phonemes in speech, can potentially be produced.

The overall advantage of this interface is that it reduces burdens on the instructor and the programmer to develop a good sound for the teaching lab. Once developed, this signal is recorded on ordinary audiotapes for the students to hear at their leisure. The students never work with the interface directly, since its only function is to replace a fistful of subroutines that would otherwise have to be written and called by the main program.

The three examples described here illustrate some of the teaching aids we've been able to develop on a fairly small computer. Situations that often boggle an instructor, such as those situations in which sensory phenomena should be demonstrated as well as studied, can often be handled without excessive efforts or costs. In summary, it seems that cost-effectiveness and ease of maintenance can be achieved in keeping with the goals of a sensation and perception laboratory by the skillful use of a computer system.

\section{NOTE}

1. A recently available General Instruments IC chip is simpler to control entirely by a parallel output port. 